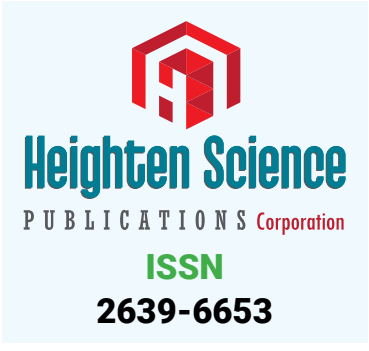

*Address for Correspondence: Dr. Hossam Al-Tatari, Chief of Pediatric Infectious Diseases, Department of Pediatrics, Tawam Hospital, Al Ain, UAE, Email: htatari@seha.ae

Submitted: 06 June 2017

Approved: 10 July 2017

Published: 11 July 2017

Copyright: @ 2017 Al Blooshi E, et al. This is an open access article distributed under the Creative Commons Attribution License, which permits unrestricted use, distribution, and reproduction in any medium, provided the original work is properly cited.

\section{Knowledge, attitude and practices associated with diagnosis and management of Skin and Soft Tissue Infections (SSTIs) among Pediatric Residents and Physicians in a Tertiary Hospital in United Arab Emirates (UAE)}

\author{
Eiman Al Blooshi ${ }^{1}$, Farah Othman ${ }^{1}$, Abeer Al Naqbi ${ }^{1}$, Majid Al \\ Rumaithi ${ }^{1}$, Khawla Fikry², Mariam Al Jneibi² and Hossam Al Tatari ${ }^{3 *}$ \\ 'Pedaitric Resident, Department of Pediatrics, Tawam Hospital, Al Ain, UAE \\ 2Intern in the Department of Academic Affairs, Tawam hospital, Al Ain, UAE \\ ${ }^{3}$ Chief of Pediatric Infectious Diseases, Department of Pediatrics, Tawam Hospital, Al Ain, UAE
}

\section{SUMMARY}

Objectives: The study aims to assess the knowledge, attitude and practices of physicians who deal with pediatric age group SSTIs in reference to the Infectious Diseases Society of America (IDSA) guidelines on this regard that was published in 2014.

Background: Skin and soft tissue infections rank among the most frequent infections worldwide.

It is estimated that over 11 million ambulatory healthcare visits occur each year in US for skin and soft tissue infections due to Staphylococcus aureus (S. aureus) alone.

SSTIs are clinical entities of variable presentation, etiology and severity that involve microbial invasion of the layers of the skin and underlying soft tissues. SSTIs range from mild infections, such as pyoderma, to serious life-threatening infections, such as necrotizing fasciitis. One of the main challenges in managing SSTIs is to be able to identify those who need immediate inpatient intervention versus the more stable ones that can be manages in outpatient basis. In June of 2014, The Infectious Diseases Society of America (IDSA) released evidence based guidelines that has nicely covered all aspects related to the management of SSTIs.

Method: An anonymous 20-item survey exploring knowledge, attitude and practices associated with diagnosis and management of skin and soft tissue infection was distributed to physicians of different levels of expertise (from residents to consultants) in the departments of Pediatrics, Emergency Medicine and Family Medicine in different governmental facilities in Al-Ain city which is one of the major cities in United Arab Emirates.

\section{INTRODUCTION}

Skin and soft tissue infections (SSTIs) rank among the most frequent infections in the pediatric age group worldwide [1]. They pose a particular predicament to pediatricians especially due to their tendency to result in significant morbidity and mortality [2,3]. The gravity of SSTI is not merely due to their primary appearance but also due to their tendency to cause blood stream infections (bacteremia, sepsis, septic shock) that are often hugely life threatening and otherwise devastating [3].

Part of the intrigue of SSTI stems from their tendency to be extremely variable in terms of presentation, etiology and degree of severity. This means that adequate knowledge of predisposing conditions, risk factors and a thorough medical history and physical examination are essential for adequacy in management of SSTI [4]. 
While the true epidemiology of SSTI is unknown considering that there are substantial numbers of people who do not seek medical attention due to the selflimiting nature of some infections. According to the Center of Disease Control (CDC) the number of hospital admissions from SSTIs increased by $29 \%$ from the year 2000 to 2004 in the United States alone [1]. Accounting for a total of 6.3 million physician office visits per year [1]. This increase in SSTIs is not limited to outpatients cases but also is apparent within hospitalized patients developing nosocomial infections. Some of this drastic increase can be attributed to the prevalence and increasing resistance of methicillin-resistant Staphylococcus aureus (MRSA) [5].

Successful treatment of SSTIs is not limited to antibiotic therapy or surgical intervention only, but is also dependent on and largely determined by the awareness of doctors, nurses, and other health care professionals of the nature and presentation of these infections [6,7]. Therefore, the purpose of this study was to assess the knowledge, attitudes, and practices on the diagnosis and management of SSTIs amongst health care practitioners across various specialties in $\mathrm{Al}$ Ain city. This was accomplished through a 16-item survey, in which questions were based on the recently revised 2014 Infectious Disease Society of America (IDSA) guidelines on the diagnosis and management of SSTIs.

\section{METHOD}

This is a cross sectional study. A hundred surveys were distributed to randomly selected physicians of different levels of expertise (from residents to consultants) in governmental facilities in Al-Ain city which is one of the major metropolitan cities in United Arab Emirates with an estimated population of about one million people. The survey included short case scenarios exploring different aspects in the management of SSTIs such indactions of admission, when to consider Pseudomonal coverage, when to order immunological work-up \& when to request surgical intervention. Informed consent was obtained from all participants.

\section{RESULTS AND DISCUSSION}

We recruited about 85 surveys for analysis (62 residents, 12 specialists, 10 consultants). $68 \%$ of the surveyed population were females and $32 \%$ were males. 48 participants were from General Pediatrics and Pediatric Intensive Care Unit (PICU), 33 from Emergency Department (ED) and 4 from Dermatology Department. The surveyed cohort had variable level of experience with $70.7 \%$ of them having less than 5 years of experience, $10.6 \%$ between $5-10$ years, $4.7 \%$ between $11-15$ years and $14 \%$ more than 15 years.

Regarding their level of comfort diagnosing SSTI, the survey revealed that $15.2 \%$ of the surveyed cohort were very comfortable diagnosing SSTIs, 57\% were comfortable, $3.5 \%$ were uncomfortable, $15.2 \%$ were very comfortable, and $2.4 \%$ were uncomfortable at all. In regard to treating SSTIs $12.9 \%$ of the surveyed cohort were very comfortable, $64.7 \%$ were comfortable, $5.8 \%$ were uncomfortable, $2.3 \%$ were uncomfortable at all.

When asked about their compliance with international SSTIs management guidelines, only 51 reported following well established international guidelines, $27 \%$ following their institute's clinical practice guidelines, $10.5 \%$ following their personal experience, $5.8 \%$ following well established national guidelines and $3.5 \%$ had no specific reference.

When asked on their opinion on current admission rates due to SSTIs, $11 \%$ of the physicians believed that admissions were always justified, $67 \%$ sometimes justified, $1 \%$ rarely, $1 \%$ never, and $20 \%$ were not sure.

The survey included twenty short case scenarios assessing physicians' knowledge and practices in managing SSTIs. They were divided into four main groups: 
Questions regarding admission criteria

Questions regarding indications of pseudomonal coverage

Questions regarding indications of immunology work-up

Questions regarding indications of surgical intervention

Figure 1 one provided details on the five questions which explored the knowledge and practices related to indications of inpatient management of SSTIs.

Significant number of participants (29.4\%) did not know that facial cellulitis must be treated in inpatient basis, while about 35.5\% did not know that a simple thigh abscess could be treated in outpatient basis. Similarly, significant number of participants $(25.6 \%)$ did not know that an infected dermoid cyst is not an indication for admission and could be treated in outpatient basis.

Never the less, when asked about the need to admit an immunocompromised patient with Central Venous Line (CVL) site infection and another with gangrenous lesion with crepitation; $98 \%$ and $94 \%$ respectively chose the right answer.

The next set of scenarios explored the participant's knowledge regarding when to consider pseudomonal coverage when managing SSTIs (Figure 2).
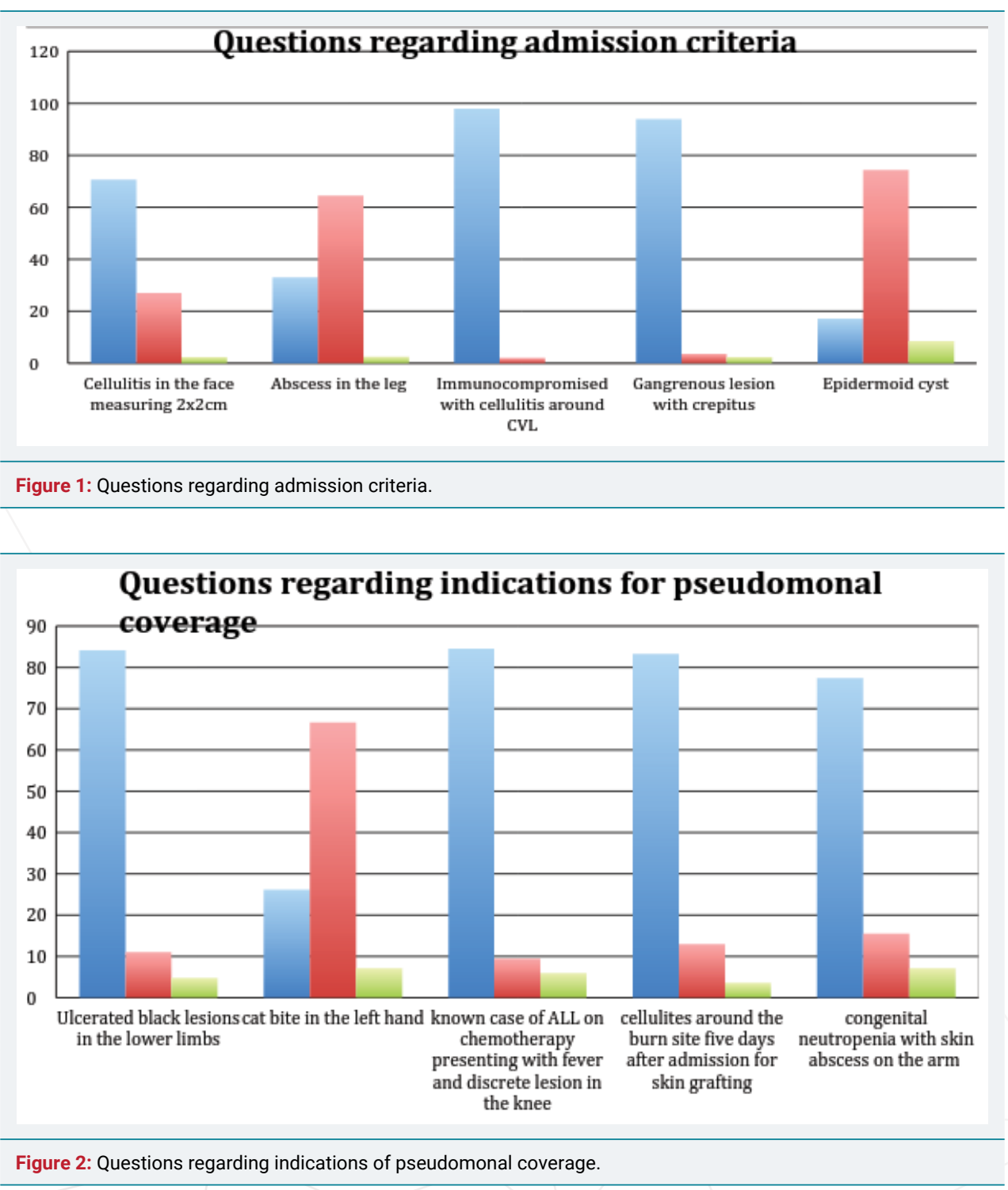
While about $84 \%$ correctly considered pseudomonal coverage in case of black ulcerated lesions and SSTIs in immunocompromised patients, still about $22.6 \%$ did not consider psedomonal coverage when treating a patient with congenital neutropenia presenting with a skin abscess. Surprisingly, about one third of participants believed -or were not sure- that pseudomonal coverage is indicated in case of cat bite.

The focus of the third set of scenarios was on the indications for immunological work up (Figure 3).

While 28.6\% would unnecessarily work up a preterm baby with recurrent CVL infections and sepsis, $9.5 \%$ of the participants would not work up a child with Asparagillus skin abscess (3.5\% were not sure) and $13.1 \%$ would not work up a child with recurrent skin abscesses $(2.4 \%$ were not sure).

The last set of scenarios were regarding indications for surgical intervention in the management of SSTIs (Figure 4).

Only in one scenario related to a complicated SSTIs in a diabetic patient, a significant number of the participants (89.3\%) correctly believed that surgical intervention is indicated. However, only $64.4 \%$ correctly recognized that nonpurulent cellulitis does not require any surgical intervention. And even a smaller
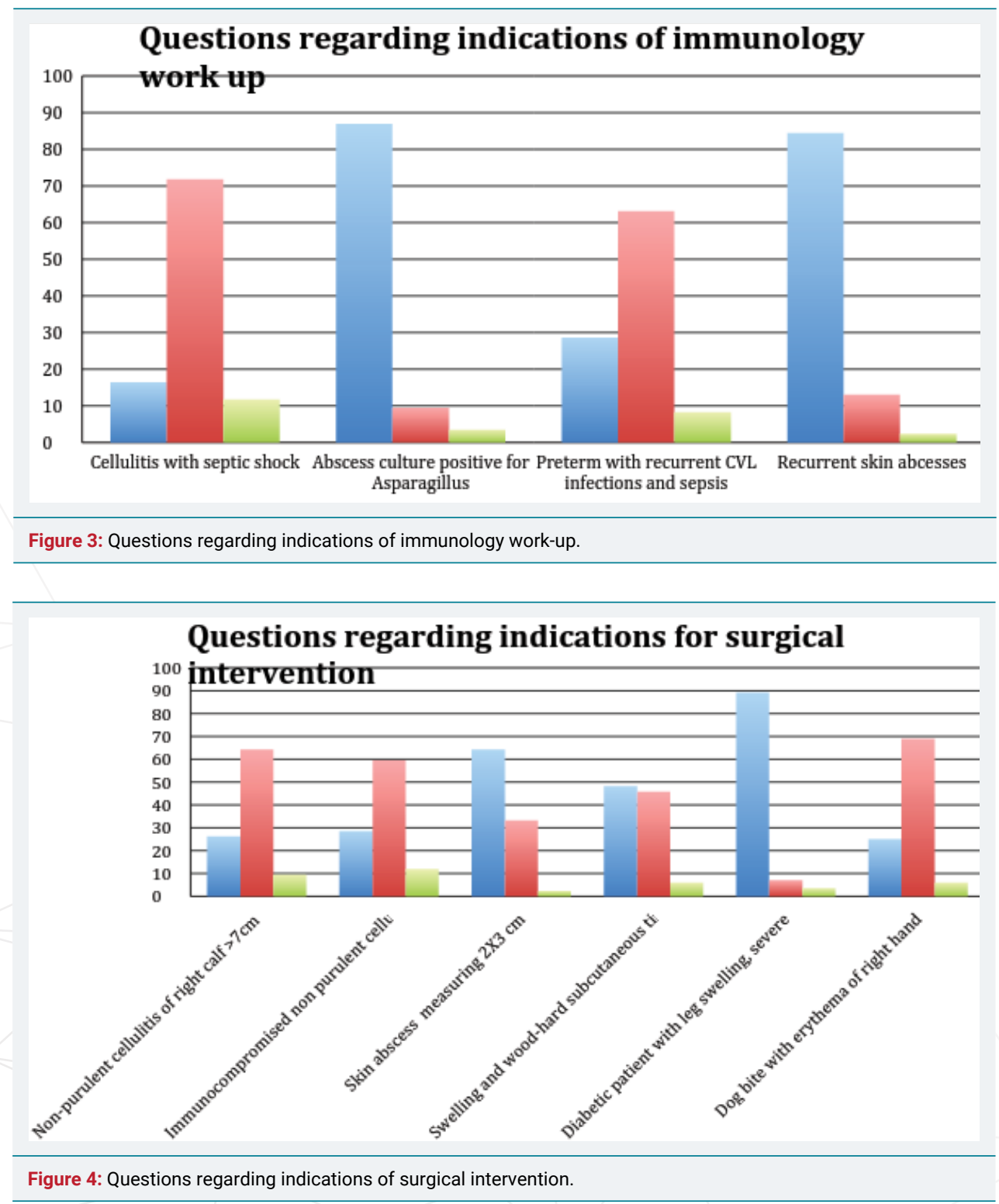
percentage of the participants (59.5\%) recognized that non-purulent cellulitis even in immunocompromised patient does not need surgical intervention. Surprisingly, more than a third of the participants did not believe that a skin abscess measuring $2 \times 3 \mathrm{~cm}$ would require surgical intervention. Also more than a half of the participants $(51.6 \%)$ did not recognize that wood hard subcutaneous tissue is a sign of necrotizing fasciitis which should be considered a surgical emergency. Finally, only $69 \%$ believed that a simple dog bite does not require surgical intervention.

\section{DISCUSSION}

The purpose of this study is to assess the knowledge, attitudes, and practices on the diagnosis and management of SSTIs amongst health care practitioners across various specialties in Al Ain city.

We included physicians who encounter skin and soft tissue infections in pediatric population frequently and participate in management plan, including physicians from general pediatrics department, PICU, emergency department and dermatology department.

The participants had different levels of experience. Most of them had less than five years of experience, which is expected since most of the participants were residents in training.

The survey identified several gaps in the knowledge and practices of the participants. Some of those gaps would result in significant morbidity and even mortality of patients. Of special significance is the fact that more than fourth did not know that a patient with facial cellulitis must be admitted for IV antibiotics since such SSTI has high risk of complications such as cavernous vein thrombosis.

Similarly, more than one fifth of the participants would not consider pseudomonal coverage in a neutropenic patient with skin abscess which could result in dissemination and poor outcome. Another alarming finding was that more than half of the participants did not recognize signs necrotizing soft-tissue infections which is a potentially lifethreatening condition characterized by rapidly advancing, local tissue destruction. Not identifying such patients and failing to provide them with emergent antibiotics administration and surgical intervention could be life threatening.

Patients harm may not only be the result of not recognizing serious conditions and managing them properly, but also due unnecessary interventions and therapies. This was another gap that was identified through some of the scenarios. For example, 33\% would unnecessarily admit a patient with a simple skin abscess and $17 \%$ would admit a patient with an infected dermoid cyst. On the other hand, more than one fourth would unnecessarily use anti-psedomonal agent when treating a cat scratch and would order an immunological work up in a premature baby with a CVL and recurrent sepsis $[8,9]$. Finally, more than one fourth of the participants would request surgical intervention in cases of non-purulent cellulitis and simple dog bites!

Although only $51 \%$ of the participants indicated that they were following well established international guidelines, still as high as $72.2 \%$ of the participants described themselves as "comfortable or very comfortable diagnosing SSTIs" and $77.6 \%$ described themselves as "comfortable or very comfortable treating SSTIs". Such a discrepancy may reflect suboptimal insight regarding the adequacy of the participants knowledge and practices. Since most of the participants are still residents in training, the above results should be an indication to include the IDSA guidelines in the residents' curriculum and to provide better training in the management of those common infections.

Up on careful review of the literature, we identified only one similar study which was conducted in Arizona, USA by Beatty, $\mathrm{N}$ et al. [10]. This study focused on the knowledge, attitudes and practices in the diagnosis and management of SSTIs among 
medical students, residents, and attending physicians in family and internal medicine at a university-based hospital in Arizona USA. In this study, a total of 103 surveys were completed. Only $35 \%$ of the participants were familiar with the updated SSTI practice guidelines provided by the IDSA. 50\% felt uncomfortable with their SSTI training and $85 \%$ felt the hospital staff would benefit from additional education. The majority (75\%) agreed that more antibiotic stewardship training is needed and $61 \%$ felt like a consultation with clinical pharmacists should be sought more often. When asked for standard treatment modalities regarding non-purulent and purulent cellulitis, 48\% answered these knowledge questions correctly. Even though 75\% of the group felt their colleagues knew when to request an appropriate surgical consult only $45 \%$ were able to recognize that this was the initial step in the management of suspected necrotizing fasciitis. $63 \%$ of the attending physicians as compared to $47 \%$ of the medical students and residents scored correctly on the knowledge section overall [10].

In comparison to our results, both studies reflects gaps in knowledge and practice toward SSTIs diagnosis and management. Those gaps were identified among those less experienced (such as residents) and those who are more expernienced (such as attendings and consultants) alike.

\section{CONCLUSION}

Although this study was limited by the relatively small sample size and the fact that most of the participants were residents in training, we still believe that the study has managed to identify several gaps in the knowledge and practices of the participating physicians in the treatment of SSTIs. While wider survey with a larger number of -and more balanced-participants may be needed, the identified gaps in knowledge and practices should be the focus of the upcoming quality improvement projects of the participating departments to provide better care in the management of SSTIs as per the IDSA guidelines.

\section{REFERENCES}

1. Sanders J, Garcia S. Evidence-Based Management of Skin and Soft-Tissue Infections in Pediatric Patients in the Emergency Department. Pediatric emergency medicine practice. 2015; 12: 1-23. Ref.: https://goo.gl/Bf8vbK

2. Low D, Teglund A. Myositis, Pyomyositis, and Necrotizing Fasciitis. Long SS, Pickering LK, Prober CG. (Eds.), Principles and practice of pediatric infectious disease. Edinburgh: Churchill Livingstone. 2012; 462-468.

3. Rausch J, Foca M. Necrotizing Fasciitis in a Pediatric Patient Caused by Lancefield Group G Streptococcus: Case Report and Brief Review of the Literature. Case Rep Med. 2011; 2011: 671365. Ref.: https://goo.gl/2hgx31

4. Dennis L Stevens, Alan L Bisno, Henry F Chambers, E Patchen Dellinger, Ellie JC Goldstein, et al. Practice Guidelines for the Diagnosis and Management of Skin and Soft Tissue Infections: 2014 Update by the Infectious Diseases Society of America. Clin Infect Dis. 2014; 59: 10-52. Ref.: https://goo.gl/1LhkWY

5. Uspal N, Agrawal D. Diagnosis and Management of Skin and Soft Tissue infections in Children. Pediatric Emergency Medicine Practice. 2008; 5: 1-28. Ref.: https://goo.gl/uS294R

6. Matiz C, Friedlander S. Subcutaneous Tissue Infections and Abscesses. Long SS, Pickering LK, Prober CG (Eds.) Principles and practice of pediatric infectious disease. Edinburgh: Churchill Livingstone. 2012; 454-462.

7. Raczniak GA, Gaines J, Bulkow LR, Kinzer MH, Hennessy TW, et al. A Survey of Knowledge, Attitudes, and Practices towards Skin and Soft Tissue Infections in Rural Alaska. Int J Circumpolar Health. 2016; 75: 30603. Ref.: https://goo.gl/7FZvwz

8. Barbara H. Pseudomonas. Baron, S. (Eds.), Medical Microbiology. Chapter 27.Galveston, Texas: University of Texas Medical Branch. 1996.

9. Dos Santos Moraes F, Antonio AG, Almeida ML, de Almeida Lima Castro R, et al. Pseudomonas Aeruginosa in the Early Childhood: A Case Report. Clin Med Case Rep. 2008; 1: 25-28. Ref.: https://goo.gl/PWVKHb

10. Beatty N, August J, Saenz JA, Nix D, Matthias K, et al. Knowledge, Attitudes, and Practices Associated With the Diagnosis and Management of Skin and Soft Tissue Infections Among Medical Students, Residents, and Attending Physicians. Open Forum Infectious Diseases. 2016; 3: 1151. Ref.: https://goo.gl/rLaR1A 